\title{
Increase in serum prolactin levels in females improves the performance of spatial learning by promoting changes in the circuital dynamics of the hippocampus
}

\author{
Beatriz Moreno-Ruiz ${ }^{a}$, Susana Mellado ${ }^{\mathrm{a}}$, Alfonsa Zamora-Moratalla ${ }^{\mathrm{a}}$, Ana L. Albarracín ${ }^{\mathrm{b}}$, \\ Eduardo D. Martín ${ }^{\mathrm{a}, *}$ \\ ${ }^{\text {a }}$ Laboratory of Neurophysiology and Synaptic Plasticity, Instituto Cajal, Consejo Superior de Investigaciones Científicas, Madrid, Spain \\ ${ }^{\mathrm{b}}$ Departamento de Bioingeniería, Instituto Superior de Investigaciones Biológicas, Consejo Nacional de Investigaciones Científicas y Técnicas, Tucumán, Argentina
}

\section{A R T I C L E I N F O}

\section{Keywords:}

Lactancy

Hyperprolactinemia

Rhythmic activity

Circuital network plasticity

\begin{abstract}
A B S T R A C T
Beyond the direct physiological functions associated with motherhood in mammals, previous studies have suggested the potential role of prolactin (Prl) in distinct brain processes such as neuroprotection, neurogenesis, and stress responses. However, the cognitive influence of Prl remains unclear, particularly regarding the mechanisms of acquisition, consolidation and retrieval of information in the brain. Using chronic implanted electrodes in freely moving female mice combined with behavioral tests, we investigated the rhythmic activity changes induced by Prl in a model of hippocampus-dependent learning and memory. Our results show that Prl improves the learning of a spatial memory task in the acquisition stage. The main variations at the circuitry level were in the theta frequency band $(4-8 \mathrm{~Hz}$ and $8-12 \mathrm{~Hz})$, marked by a faster change in oscillatory activity with no modifications to higher frequencies. These results show that Prl plays a significant role in the acquisition of information during learning of a spatial memory task, suggesting that an increase in Prl levels may induce changes in circuital network plasticity.
\end{abstract}

\section{Introduction}

Prolactin (Prl) is a peptide hormone that is primarily synthesized and secreted by lactotrophic cells in the adenohypophysis, but likewise by extrapituitary tissues (Grattan and Kokay, 2008). The broad spread of $\mathrm{Prl}$ and its receptor leads to diverse physiological functions in mammals, from reproduction to metabolism, growth and development, osmoregulation, immune function, lactogenesis and motherhood behavior (Bole-Feysot et al., 1998; Bridges and Grattan, 2019). Interestingly, Prl may be involved in various brain processes (Bridges and Grattan, 2019), and there is clinical and experimental evidence that supports the role of Prl in cognition (Henry and Sherwin, 2012; Walker et al., 2012; Torner et al., 2013; Bala et al., 2016; Yao et al., 2018). For example, impairment in tasks of verbal recall and processing speed during late pregnancy and the early postpartum period has been associated with high levels of Prl and steroid hormones (Henry and Sherwin, 2012). The hippocampus is an important brain area involved in cognitive functions like spatial information acquisition as well as retrieval and storage of memory (Barker and Warburton, 2011). In line with this, experimental evidence indicates that the combination of pregnancy and motherhood enhances performance in hippocampus-dependent memory tasks (Pawluski et al., 2006), and reproductive experience significantly improves spatial reference and working memory (Kinsley et al., 1999; Love et al., 2005; Pawluski et al., 2006). However, the direct effect of Prl has not been clearly identified, and reports that indicate the participation of Prl in learning and memory are limited. For instance, Prl-null mice showed significant hippocampal-dependent learning and memory deficits that were restored with the infusion of Prl directly in the hippocampus (Walker et al., 2012). On the other hand, hyperprolactinemia in male rats impaired object recognition without altering spatial learning (Torner et al., 2013). In addition, regular exercise protected hippocampal-dependent memory consolidation during chronic stress by improving Prl responsiveness (Leem et al., 2019). Therefore, the specific role of Prl in hippocampal-dependent learning and memory and their mechanisms at circuital level remain elusive. We hypothesize that Prl influences performance in memory tasks and consequently the underlying circuital plasticity. To address this issue, in the present study we evaluated the interaction between Prl and behavior, analyzing the

\footnotetext{
* Corresponding author at: Instituto Cajal, CSIC, Av. Doctor Arce 37, 28002, Madrid, Spain.

E-mail address: eduardo.martin@cajal.csic.es (E.D. Martín).
} 
neuronal oscillations that underlie the processing of information.

\section{Materials and methods}

\subsection{Animals}

Female C57BL/6JRj mice (8-12 weeks old) were housed in groups under a standard 12:12 h light/dark cycle with access to food and water ad libitum. All animal-related procedures were in accordance with European (2010/63/UE) and Spanish (RD 53/2013) regulations regarding animal welfare and were approved by the institutional committee for ethics and animal use (Cajal Institute Ethics Committee on Human and Animal Experimentation and CSIC Ethics Committee).

\subsection{Experimental protocol}

Female mice were daily injected subcutaneously (s.c.) with $200 \mu \mathrm{g}$ of ovine Prl from sheep pituitary (Sigma-Aldrich, St. Louis, MO, USA) dissolved in vehicle $(0.9 \% \mathrm{NaCl})$ or with vehicle alone $(\mathrm{Vh})$. The treatment started three days before the beginning of the memory task and lasted until the day before the long-term trial on day 8 (10 total days of treatment).

\subsection{Electrode implantation}

Mice were placed in a stereotaxic frame (Kopf Instruments, Model 902, Tujunga, CA, USA) under isoflurane anesthesia (3\% for induction and $0.5-1 \%$ for maintenance). Two isolated stainless steel electrodes $(50-80 \mu \mathrm{m})$ and one silver electrode as ground were assembled in a four-channel lightweight miniature socket that was fixed to the skull with stainless steel screws and dental cement. Electrode implantation was performed according to the stereotaxic atlas (Paxinos and Franklin, 2012) with reference to bregma. Two electrodes were lowered into each hemisphere along the septo-temporal axis of the dentate gyrus of the dorsal hippocampus ( $-2 \mathrm{~mm} \mathrm{AP},+1.4 \mathrm{~mm} \mathrm{ML}, 1.7-1.9 \mathrm{~mm}$ DV, Fig. 1A, left). The reference electrode was positioned in the right motor cortex $(-1 \mathrm{~mm} \mathrm{AP},+1.5 \mathrm{~mm} \mathrm{ML}, 1 \mathrm{~mm} \mathrm{DV})$ and the silver electrode (ground) was located on the surface of the left prefrontal cortex $(+1.7 \mathrm{AP},+0.3$ $\mathrm{mL}$ ). Following surgery, the animals were allowed to recover for at least one week prior to further experimental protocols. The location of the electrode tips was determined postmortem by immunochemistry (Fig. 1A, left).

\subsection{Electrophysiology}

Hippocampal electrophysiological recordings were carried out with a 4-channel wireless system device (W4-Headstage, Multichannelsystems MSC GmbH, Reutlingen, GE,) synchronized with a video camera. Recordings were digitized at $20 \mathrm{KHz}$ and bass-pass filtered at $200 \mathrm{~Hz}$ (Butterworth 2nd order filter). For spectral analysis, a fast Fourier transform employing $2 \mathrm{~s}$ bins was performed using a Hamming window with $50 \%$ overlap (Clampfit 10.2, Axon Instruments, Molecular Devices Corporation, Sunnyvale, CA, USA) to analyze the correlation between hippocampal oscillations and precise activity during the behavioral task. Since animals had different levels of baseline power density $\left(\mathrm{V}^{2} . \mathrm{Hz}^{-1}\right)$, the power density (PD) values for each frequency were normalized as a percentage of the total power density recorded before computing group results. The frequency bands selected were: $3-8 \mathrm{~Hz}, 8-12 \mathrm{~Hz}$ and 65-100 Hz (Fig. 1A, right). The average speed and power density in the different frequency bands were correlated with the period between the beginning of the task and arrival to the target hole in the Barnes maze (BM). The cross correlation frequency estimation (CCFE) was calculated for grouped frequencies in bins of $1 \mathrm{~Hz}$ from 3 to $19 \mathrm{~Hz}$ or $65-100 \mathrm{~Hz}$ (Clampfit 10.2) and values were normalized with respect to the theta power density on D1 (intragroup analysis) or between conditions (i.e. vehicle vs. Prl) on the same day (cross-group analysis). Since significant differences were found at D2 between experimental conditions (see Results), we focused our analysis on D2 (acquisition), D3 (consolidation) and D5 (spatial memory retention).

\subsection{Barnes maze}

7 days after surgery the animals were trained in the BM (Barnes, 1979), which consists of a circular platform (92-cm diameter) elevated
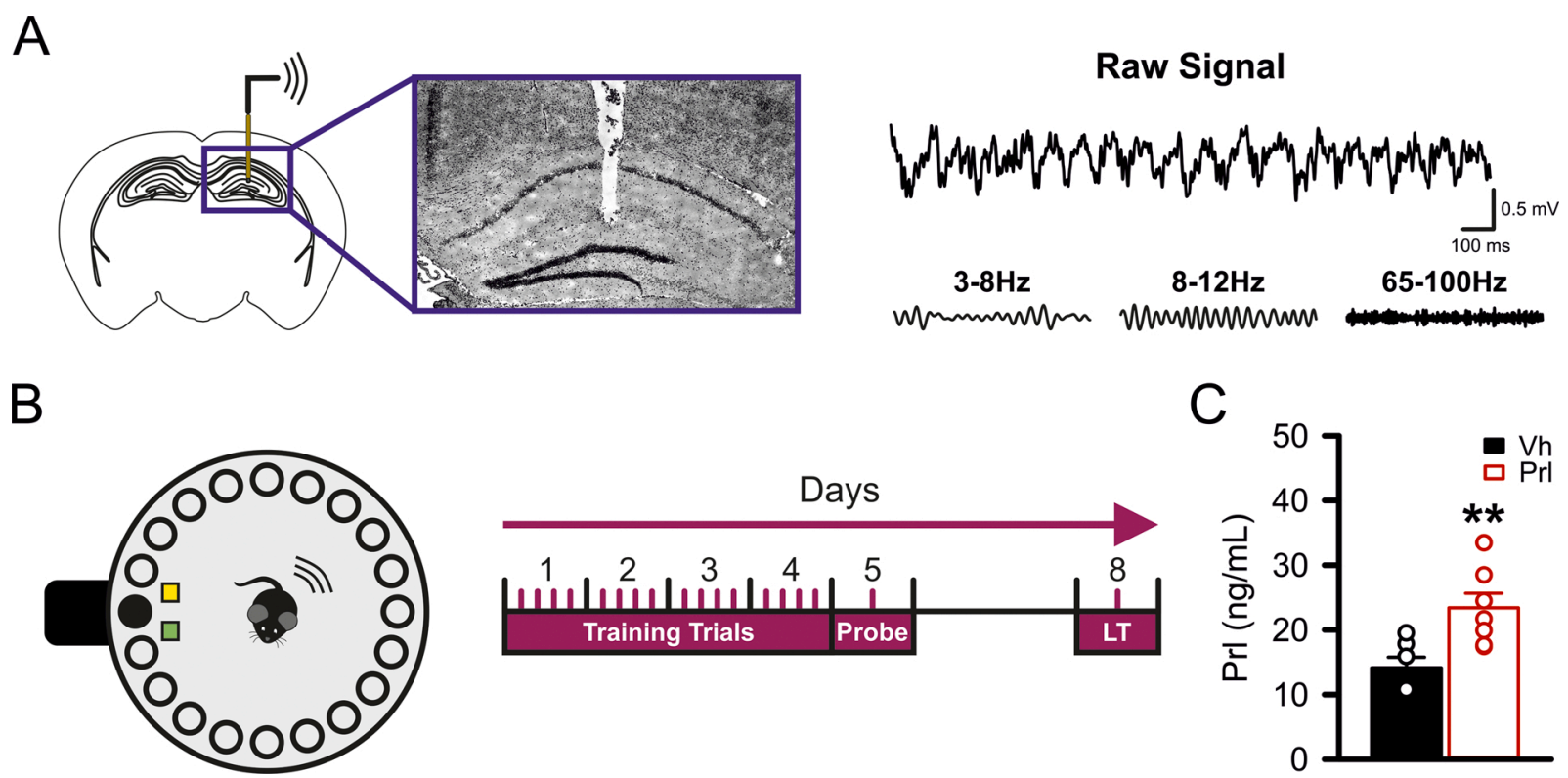

Fig. 1. Experimental procedures. (A) Hippocampal recordings were performed using a wireless system during training (left). Inset is a representative Hoechststained brain section showing a track corresponding to an electrode implanted in the dorsal hippocampus. Right, typical recording of oscillatory activity while the animals performed the maze. Raw signal was filtered in the frequency bands of 3-8 Hz, 8-12 Hz and 65-100 Hz. (B) Schematic representation of the Barnes Maze (BM) and schedule of the training. The treatment began 3 days before D1 and lasted 10 days, during which female mice received saline or prolactin. (C) Summary data showing mice serum prolactin levels (ng/mL) after treatment with vehicle (Vh, black filled bar) and prolactin (Prl, red empty bar). Significant differences were established at ${ }^{* *} \mathrm{p}<0.01$ between $\mathrm{Vh}$ - and Prl-treated animals $(\mathrm{Vh} \mathrm{n}=6$, Prl $\mathrm{n}=7$ ). Error bars represent s.e.m. 
$100 \mathrm{~cm}$ above the floor with 20 equidistant holes $(5 \mathrm{~cm}$ in diameter separated by $7.5 \mathrm{~cm}$; Fig. 1B, left). In the maze, 19 holes were closed while under the "escape hole" there is a box $(23 \times 15 \times 9 \mathrm{~cm})$ as a reinforcement. The BM was placed in a dark room where an intense light in the center encouraged the mice to escape. External and internal cues to the maze remained at a fixed position during the complete performance. Prior to training, animals were habituated to the testing room for $10 \mathrm{~min}$. Mice were trained for 4 days, with 4 trials per day and an inter-trial interval of $15 \mathrm{~min}$ (Fig. 1B, right). Between trials, the maze was wiped with $70 \%$ ethanol to avoid olfactory cues. Animals were allowed to freely explore the platform and find the escape box for $3 \mathrm{~min}$. If the mouse did not find the target in the given time, it was then guided to the hole. Spatial memory retention was tested $24 \mathrm{~h}$ after the last training trial (day 5). To assess long-term retention, a probe trial was applied on day 8, without any training session between day 5 and day 8 (Fig. 1B, right). To monitor the learning and motor performance, the following parameters were considered:

a) Number of holes checked before mouse escape into the target hole.

b) Latency to locate the target hole.

c) Total distance traveled to locate the target hole.

d) Average speed reached to cover the distance between the center of the maze and the target.

e) Time spent around the cues and near the target hole (distraction time) in order to calculate a distraction/latency ratio.

\subsection{Immunohistochemistry}

To end the experiments, animals were deeply anesthetized by intraperitoneal injection of ketamine/xylazine $1: 1(0.1 \mathrm{~mL} / \mathrm{kg})$, and transcardially perfused with saline $(25 \mathrm{~mL})$ followed by $4 \%$ paraformaldehyde (PFA; $100 \mathrm{~mL}$ ) pH 7.4 in $0.1 \mathrm{M}$ phosphate buffered saline (PBS). Brains were dissected and post-fixed in PFA for $4-8 \mathrm{~h}$ at $4{ }^{\circ} \mathrm{C}$, immersed in $30 \%$ sucrose for $48 \mathrm{~h}$ and embedded in tissue freezing medium (Neg-50, Thermo Fisher Scientific) by freezing in liquid nitrogen. Transversal cryosections were cut at $20 \mu \mathrm{m}$ using a Microm HM 550 cryostat (Thermo Fisher Scientific, Waltham, USA) and collected on SuperFrost Plus slides (Thermo Fisher Scientific, Waltham, USA). After permeabilization with $0.2 \%$ Triton X-100 in phosphate buffered saline (PBS) $2 \mathrm{~h}$ at room temperature (RT), nuclear staining was performed with Hoechst (1:5000; \#B2261; Thermo Fisher Scientific, Waltham, USA). Samples were extensively washed with PBS, and images were acquired on an LSM 800 confocal microscope (Zeiss, Oberkochen, Germany).

\subsection{Measurement of prolactin}

Blood was collected from the submandibular vein and centrifuged at $2000 \mathrm{xg}$ for $5 \mathrm{~min}$, and serum was collected and stored at $-80^{\circ} \mathrm{C}$. Prl concentrations were quantified by a prolactin mouse ELISA Kit (Abcam ab100736), following the manufacturer's instructions. Briefly, 1) $100 \mu \mathrm{L}$ of each standard and sample was added into wells and incubated $2.5 \mathrm{~h}$ at RT; 2) the solution was discarded and washed 4 times with $1 \mathrm{x}$ Wash Solution (300 $\mu \mathrm{L})$; 3) $100 \mu \mathrm{L}$ of 1x Biotinylated Prl Detection Antibody was added and incubated for $1 \mathrm{~h}$ at RT with gentle shaking; 4) The solution was discarded and washed 3 times; 5) $100 \mu \mathrm{L}$ of $1 \mathrm{X}$ HRPStreptavidin solution was added and incubated for $45 \mathrm{~min}$ at RT with gentle shaking; 6) The solution was discarded and washed 3 times; 7) $100 \mu \mathrm{L}$ of TMB One-Step Substrate Reagent was added and incubated for 30 min at RT in the dark with gentle shaking; 8) $50 \mu \mathrm{L}$ of Stop solution was added and then absorbance values were read at $450 \mathrm{~nm}$ in a plate reader (Bio-Rad).

\subsection{Statistical analysis}

Appropriate statistical analysis was chosen by the normality of the data and expressed as mean \pm standard error of the mean (s.e.m.). Statistical analyses were performed using SPSS software. Based on the distribution of the data for independent measures, the level of significance was determined using parametric (Student's t test) or nonparametric (Mann-Whitney U) tests. For the linear regression, a fitting model was used. For all analyses, values of $\mathrm{p}<0.05$ were considered statistically significant.

\section{Results}

There is evidence to support that learning and the performance of memory tasks are influenced by Prl (Walker et al., 2012; Torner et al., 2013). However, the role of high levels of Prl in the functional circuital modifications that underlie hippocampal-dependent learning and memory have not been completely clarified. To address this issue, we analyzed the behavior of control (Vh) and Prl (200 $\mu$ g s.c./day)-treated animals in the BM, a useful task to assess spatial reference memory in mice (Sunyer et al., 2007). Daily treatment with $200 \mu \mathrm{g}$ of Prl yielded a significant increase in serum Prl level (Student's t-test: $\mathrm{t}_{11}=-3.222, p<$ 0.01, Vh $n=6, \operatorname{Prl} n=7$; Fig. 1C). Across training days (Fig. 2A, left panel), a statistically significant decrease in the number of errors before mice escaped into the target hole (Fig. 2A, center panel) was observed in the acquisition phase (day 2 of the task; D2) in mice treated with Prl compared to Vh (Mann-Whitney U test: $\mathrm{U}=20.5, p<0.01, \operatorname{Prl} n=11$, Vh $n=11$, Fig. 2A, center panel), showing significant differences in the repetitions of trials 3 (Mann-Whitney $\mathrm{U}$ test: $\mathrm{U}=26.5, p<0.05, \operatorname{Prl} n=$ $11, \mathrm{Vh} n=11$, Fig. 2A, right panel) and 4 (Mann-Whitney $\mathrm{U}$ test: $\mathrm{U}=23$, $p<0.05, \operatorname{Prl} n=11$, Vh $n=11$, Fig. 2A, right panel) on D2. However, no Prl effect was observed in the short- or long-term trial (see D5 and D8 respectively; Fig. 2A, center panel). In addition, behavioral measurements such as latency, distance or speed were similar in both groups (Fig. 2B), indicating that Prl-treated mice make fewer errors but require the same amount of time to solve the maze as the control mice. To address this question, we measured the "distraction time" (see Methods) to calculate the distraction/latency ratio on D2. We found that Prl-treated animals employed $18.97 \%$ of the time included in latency exploring around the cues and close to the target, whereas in Vh-treated animals this time was $2.15 \%$ (Fig. 2B, right panel). Taken together, these data indicate that Prl improves the learning performance of a spatial memory task in the acquisition phase.

To investigate the functional circuital modifications that underlie the observed behavioral changes, we analyzed the correlation between power density of the signal per frequency (Fig. 3A) and arrival at the target on D2 (acquisition), D3 (consolidation) and D5 (spatial memory retention) and the rest phase of task acquisition (Fig. 3B, upper panels). The power of rhythmic activity measured in Vh- and Prl-treated mice was similar at all frequencies upon arrival on D2 (Fig. 3B, lower panels). Interestingly, analysis of the distribution of power across frequency bands at rest on D2 revealed a significant decrease in the $3-8 \mathrm{~Hz}$ band of Prl-treated animals (Student's t-test: $\mathrm{t}_{15}=2.272 ; p<0.05$, Vh $n=8$. Prl $n=9$; 3B, lower panels) that correlated to an increase in $8-12 \mathrm{~Hz}$ (Mann-Whitney U test: $\mathrm{U}=65 ; p<0.01$, Vh $n=8$. $\operatorname{Prl} n=9$; 3B, lower panels). These significant differences were maintained between $3-8 \mathrm{~Hz}$ (Student's t-test: $t_{8}=2.994 ; p<0.05$, Vh $n=8$. Prl $n=9$; 3B, lower panels) and 8-12 Hz (Mann-Whitney U test: $\mathrm{U}=60 ; p<0.05$, Vh $n=8$. $\operatorname{Prl} n=9$; 3B, lower panels) in the arrival on D3 but only in the $8-12 \mathrm{~Hz}$ band in the arrival on D5 (Student's t-test: $\mathrm{t}_{15}=-2.441 ; p<0.05$, Vh $n=$ 8. $\operatorname{Prl} n=9$; 3B, lower panels). In addition, no changes in the power of gamma frequencies were observed in either experimental condition at different stages of BM task acquisitions (3B, lower panels). These data indicate that an increase in serum Prl modulates hippocampal theta rhythmic activity during spatial learning, which might be involved in cognitive performance.

Since previous studies have shown that changes in $\theta$ oscillations are related to the speed of animals in different tasks (Rivas et al., 1996; Bender et al., 2015), we next examined the relationship between the 


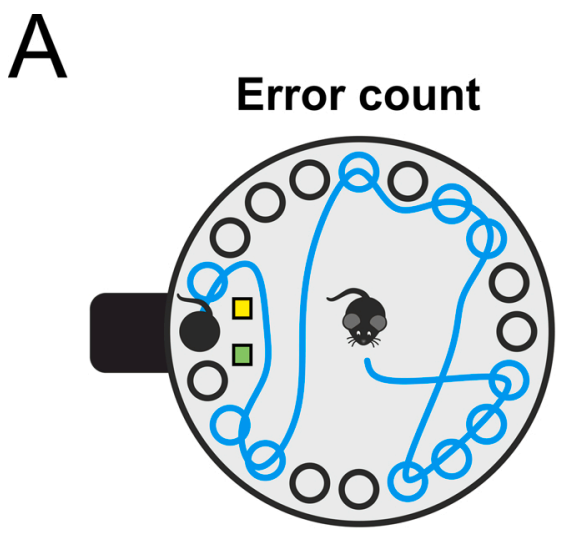

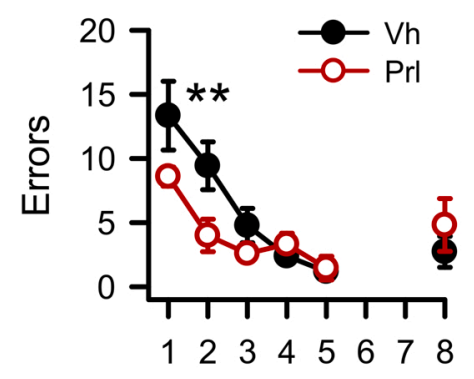

Day

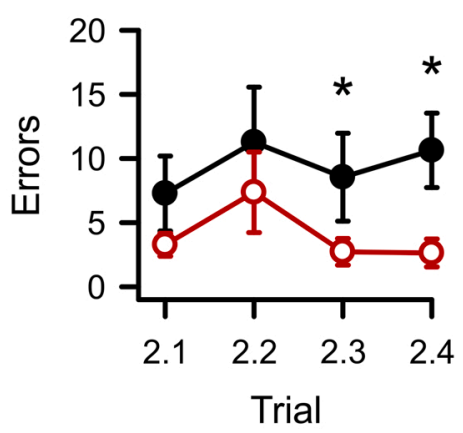

Trial

B

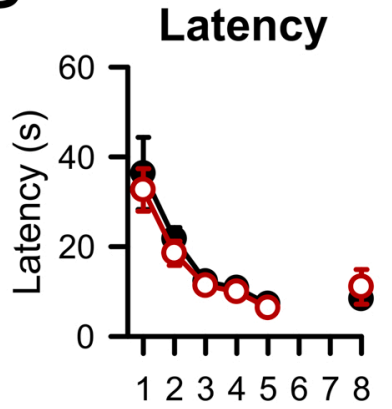

Day
Distance

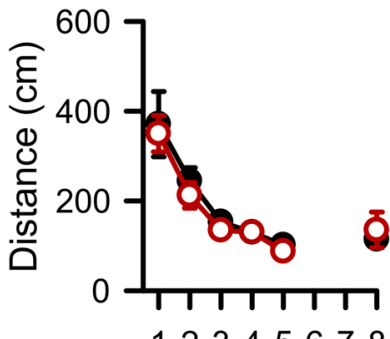

12345678

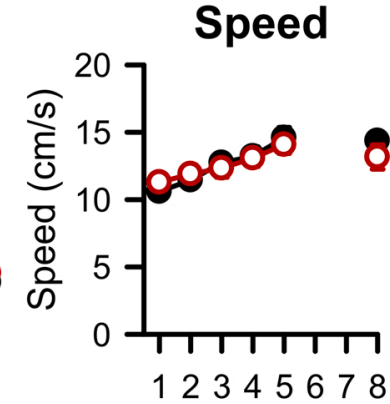

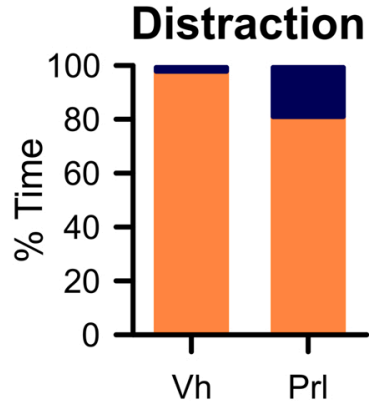

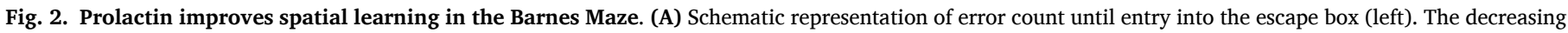

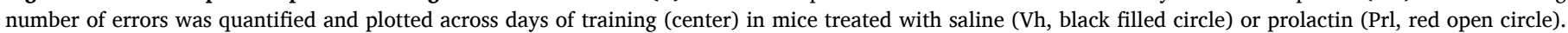

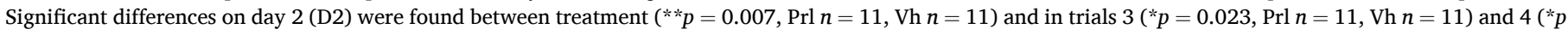

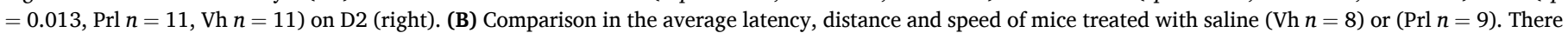

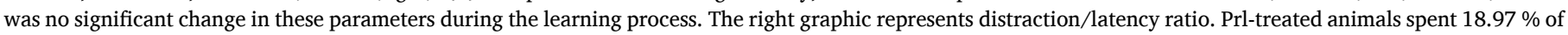
the time (blue) included in latency (orange) exploring, whereas Vh-treated mice spent $2.15 \%$. Error bars represent s.e.m.

speed and PD of the different frequency bands. No statistical significance was found between Vh- and Prl-treated animals either on D3 (Student's t-test; $3-8 \mathrm{~Hz}: \mathrm{Vh} n=8, p>0.05 ; \operatorname{Prl} n=9, p>0.05 ; 8-12 \mathrm{~Hz}: \mathrm{Vh} n=8$, $p>0.05 ; \operatorname{Prl} n=9, p>0.05 ; 65-100 \mathrm{~Hz}: \operatorname{Vh} n=8, p>0.05 ; \operatorname{Prl} n=9, p$ $>0.05$; Fig. 4 A) or on D5 ( $3-8 \mathrm{~Hz}$ : Vh $n=8, p>0.05$; $\operatorname{Prl} n=9, p>$ 0.05; 8-12 Hz: Vh $n=8, p>0.05 ; \operatorname{Prl} n=9, p>0.05 ; 65-100 \mathrm{~Hz}: \mathrm{Vh} n$ $=8, p>0.05 ; \operatorname{Prl} n=9, p>0.05$; Fig. 4B), indicating that the differences observed in rhythmic activity from 3 to $12 \mathrm{~Hz}$ were not due to changes in speed resulting from the treatment.

A more detailed analysis of the distribution of power across frequency bands indicated that during arrival on D2 (intragroup analysis, Fig. 5A) there was a shift in lower oscillations $(7-9 \mathrm{~Hz})$ with respect to D1 in both Vh- and Prl-treated animals (Fig. 5A, $\theta-\beta$ ), which was not evident between conditions on the same day (D2; Fig. 5A, $\theta-\beta$ ). This change was most clear when performing a cross-correlation analysis of different frequencies in the $\theta$ band (Fig. 5A, $\theta$-CCFE). Furthermore, we observed a higher PD percentage in Prl-treated mice at $9 \mathrm{~Hz}$ (Fig. 5A, $\theta-\beta)$. In $\gamma$ frequencies, a decrease was observed in the D2, D3 and D5 arrival PD percentage relative to $\mathrm{D} 1$, with a similar synchronic activity pattern (Fig. 5A, C and D, $\gamma$ ) that was confirmed by a cross-correlation analysis between days in the $\gamma$ band (Fig. 5A, C and D, $\gamma$-CCFE). However, no significant differences were found between the animals treated with Prl compared to controls. During rest in the escape box, the shift in the Prl-treated group was maintained with a widening of the $\theta$ band (Fig. $5 \mathrm{~B}, \theta-\beta$ ), with a clear difference in the cross-correlation analysis compared to Vh-treated animals (Fig. 5B, $\theta$-CCFE). In addition, no difference was observed between groups in the $\gamma$ band during rest in the escape box (Fig. 5B, $\gamma$ ). Interestingly, the shift at lower oscillations and the higher PD percentage at $9 \mathrm{~Hz}$ that were observed during arrival between D1 and D2 were maintained between D1-D3 and D1-D5 (Fig. 5C and $\mathrm{D}$, respectively). Taken together, these data suggest a faster change in the low oscillatory activity during rest on D2, underlying the consolidation of spatial information in the hippocampus during acquisition of the learning course.

\section{Discussion}

Beyond the well-established effects of Prl in physiological and pathological conditions, cumulative evidence over the past few decades indicate that this pleiotropic hormone is able to induce relevant changes in several brain process (Bridges and Grattan, 2019; Cabrera-Reyes et al., 2017). In line with this, various experimental evidence suggests that Prl per se may modify hippocampal function (Walker et al., 2012; Torner et al., 2013; Leem et al., 2019), but the specific role of Prl in the brain's capacity to acquire spatial information, storage and retrieval of memory remains elusive. To address this issue, we used an experimental approach to simultaneously analyze a spatial memory task and hippocampal rhythmic activity as an indicator of the functional circuital modifications that underlie the observed behavioral changes. In addition, we doubled normal serum Prl levels in order to obtain a moderate degree of hyperprolactinemia. In these experimental condition, Prl enhanced performance of a spatial memory task, in agreement with previous results (Walker et al., 2012; Leem et al., 2019). This effect was more evident on D2 (acquisition phase), particularly during the 3rd and 4th trial of the day. However, other behavioral measurements such as latency, distance or speed were similar in both groups, indicating that 
Raw Signal

Vh

Prl

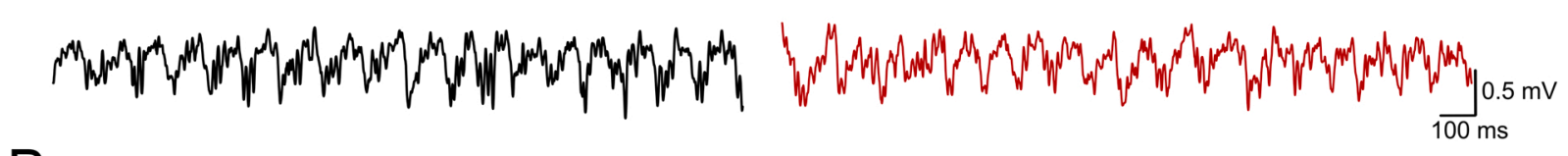

B

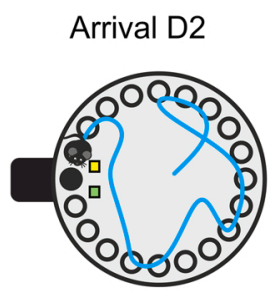

Grouped $\theta-\gamma$

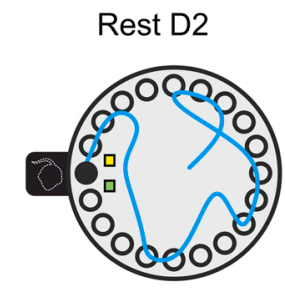

Grouped $\theta-\gamma$
Arrival D3

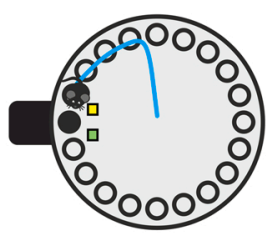

Arrival D5

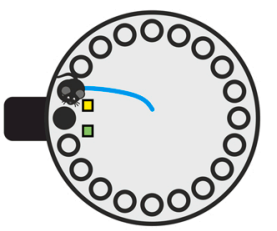

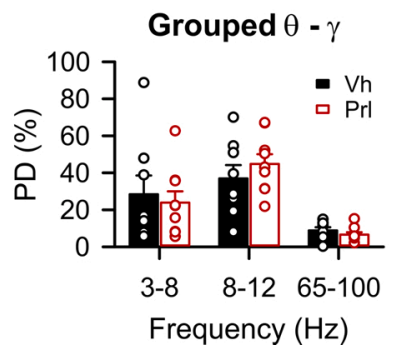
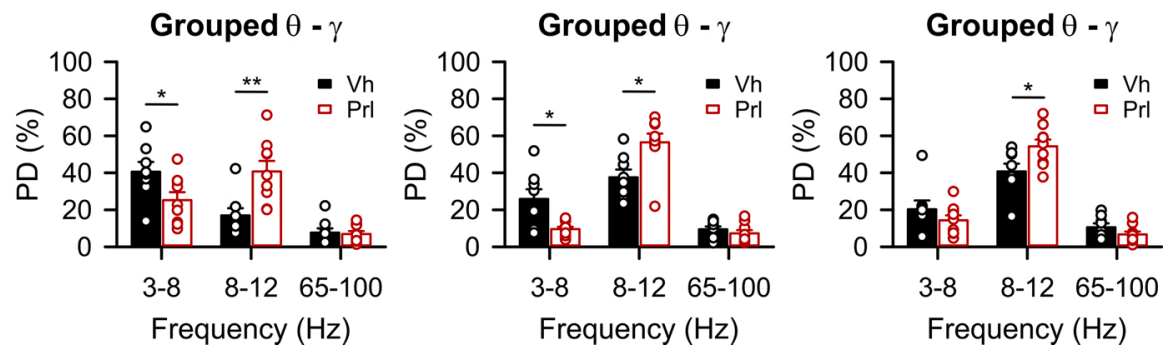

Fig. 3. Changes in the rhythmic activity of the hippocampus underlie the improved performance of prolactin-induced spatial learning. (A) Typical recordings of raw signals in the dentate gyrus of the hippocampus $2 \mathrm{~s}$ before the arrival to the maze exit in mice treated with saline (Vh, left) or prolactin (Prl, right). (B) Mean power density of the $\theta-\gamma$ band of mice treated with vehicle (Vh, black filled) or prolactin (Prl, red empty) upon arrival and rest on D2 (acquisition) and arrival on D3 (consolidation) and D5 (spatial memory retention). Power density (PD) in the $\theta$ band was significantly different between Vh- and Prl-treated mice during rest on D2 (3-8 Hz, ${ }^{*} p=0.038 ; 8-12 \mathrm{~Hz},{ }^{*} p=0.004$, Vh $n=8$. $\left.\operatorname{Prl} n=9\right)$; upon arrival on D3 (3-8 Hz, ${ }^{*} p=0.018 ; 8-12 \mathrm{~Hz},{ }^{*} p=0.021$, Vh $n=8$. Prl $\left.n=9\right)$ and arrival on D5 (8-12 Hz, ${ }^{*} p=0.028, \operatorname{Vh} n=8$. $\left.\operatorname{Prl} n=9\right)$. No difference was found in the power density of $\gamma$ range. Each dot represents a single animal. Error bars represent s.e.m.

A

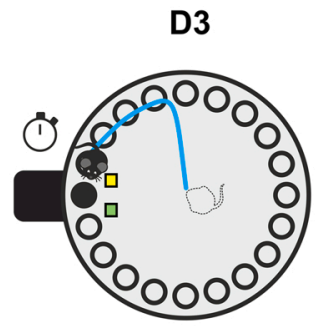

B

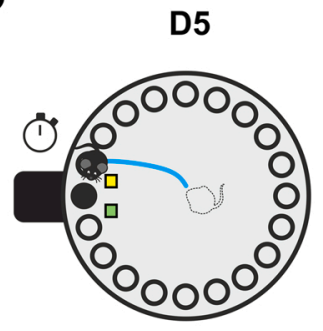

$3-8 \mathrm{~Hz}$
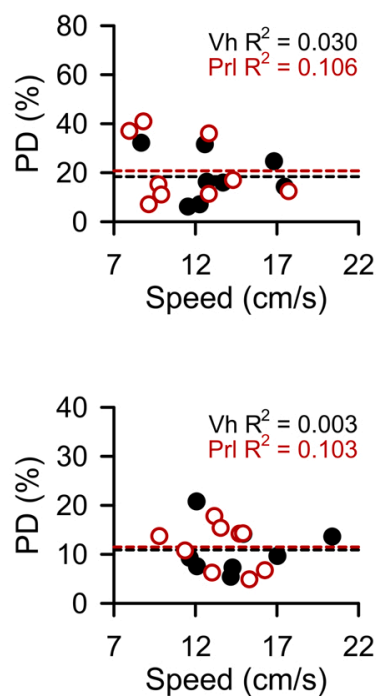

8-12Hz
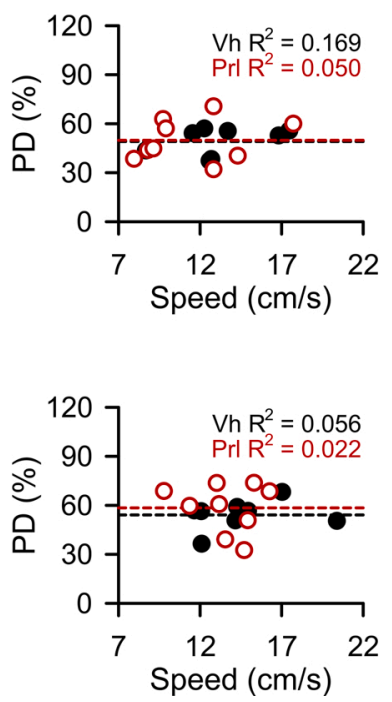

$65-100 \mathrm{~Hz}$
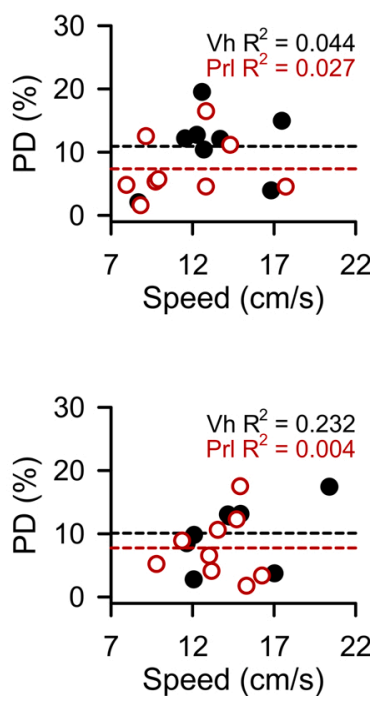

Fig. 4. Speed-frequency correlation on consolidation and spatial memory retention days. (A) Linear relationship between $\theta-\gamma$ band power density (PD) and speed on day 3 (D3). No significant change was found in control (Vh) vs. prolactin (Prl)-treated animals across the $\theta-\gamma$ band (Vh $n=8$, Prl $n=9$ ). (B) Same as (A) but on day 5 (D5) for 3-8 Hz, 8-12 Hz and $65-100 \mathrm{~Hz}(\operatorname{Vh~} n=8, \operatorname{Prl} n=9)$ frequency bands. Each dot represents a single animal.

Prl-treated animals invested the same amount of time solving spatial memory tasks as the control mice. Interestingly, during performance of the task, Prl-treated animals stopped more frequently and spent more time around the spatial internal cues and exploring close to the target than Vh-treated animals, even when they were oriented directly to the target. As a result, a difference in the number of errors was observed but 
A

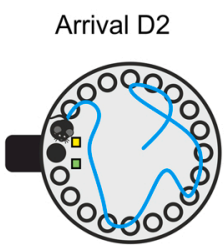

$\mathrm{B}$

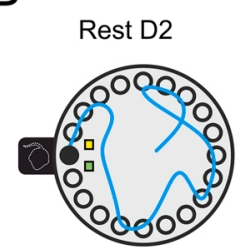

C

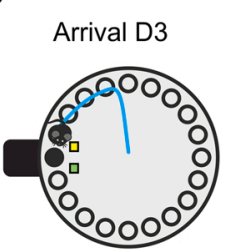

D

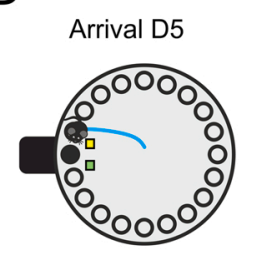

$\theta-\beta$
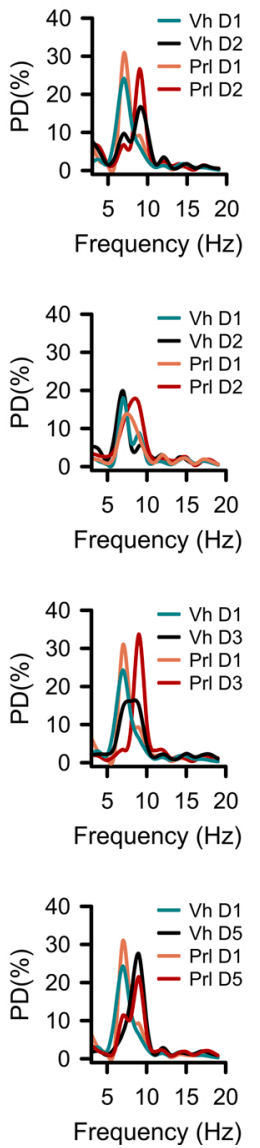

$\theta$ - CCFE
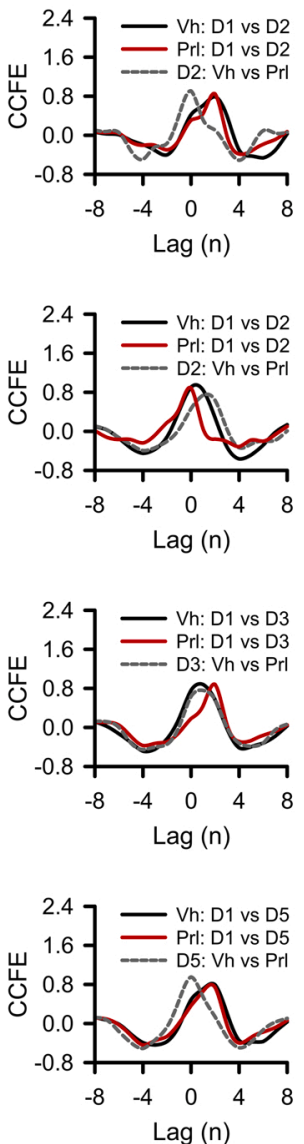

$\gamma$
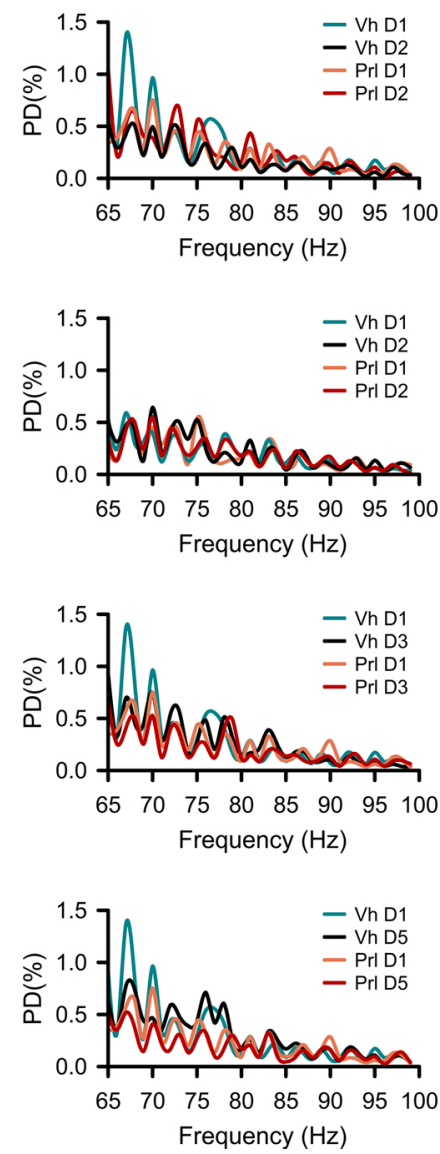
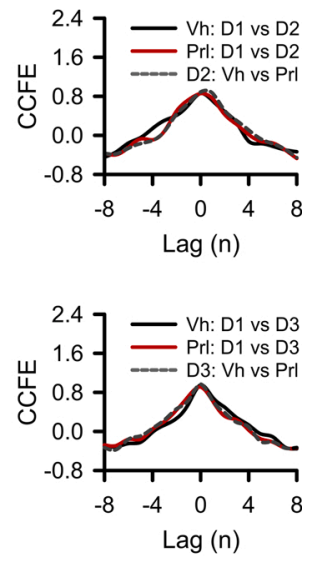

$\gamma$ - CCFE
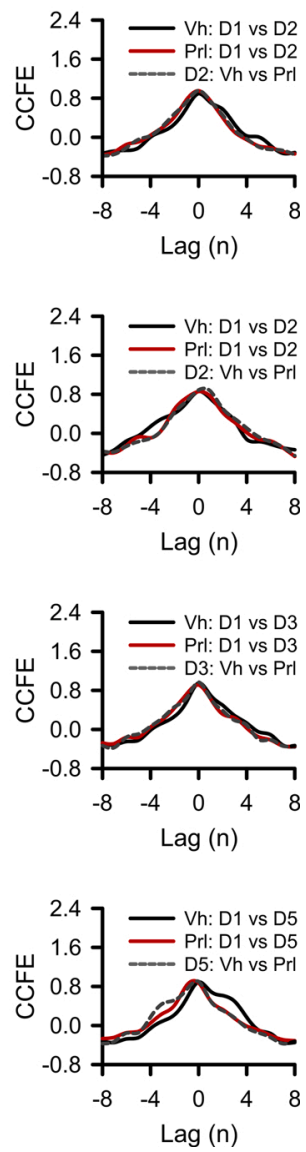

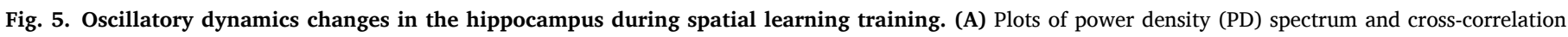

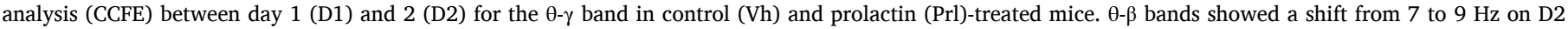

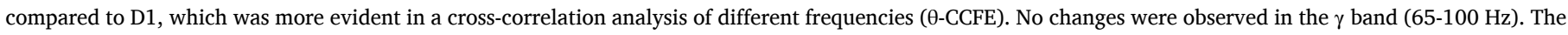

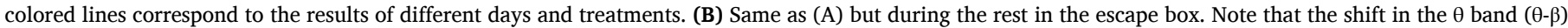

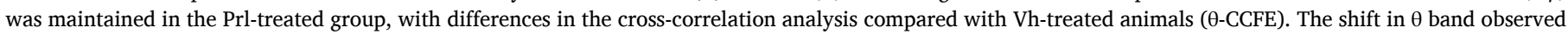
during arrival between D1 and D2 was maintained between D1-D3 (C) and D1-D5 (D) without changes in the $\gamma$ band (65-100 Hz).

not in latency or distance. Future studies, beyond the scope of present work, will be necessary to elucidate this curious behavior in Prl-treated animals. In any case, analyzing the data as a whole, our results reinforce the idea that increased serum levels of this hormone per se can influence hippocampal-dependent skills.

Learning and memory imply a structural and functional remodeling at synaptic and circuital levels (Martin et al., 2000; Nicoll, 2017; Segal, 2017). In this way, brain rhythms (particularly hippocampal oscillations) are necessary to compute behaviorally relevant information, representing the temporal frame in which memories are encoded (Winson, 1978; McNaughton et al., 2006). Performance in spatial memory tasks depends on hippocampal activity (Barker and Warburton, 2011), and neuronal oscillations in the dentate gyrus change with training in the BM (Bott et al., 2016). Specifically, a learning-dependent increase in theta-gamma coupling has been detected near the target area of BM (Bott et al., 2016). We observed a decrease in the $3-8 \mathrm{~Hz}$ band of Prl-treated animals that was associated with an increase at $8-12 \mathrm{~Hz}$ which was statistically significant during rest on D2, coinciding with the day of Prl-dependent performance increase. However, no differences in frequency bands were found on D2 during trial sessions or near the target area during BM testing. This evidence may indicate that changes in hippocampal rhythmic activity, which underlie the behavioral modification induced by Prl, occur in a short temporal window after trial in the rest of acquisition phase. Interestingly, differences in theta oscillations were maintained on arrival at D3 and only in the $8-12 \mathrm{~Hz}$ band on arrival at D5 (retention phase), even though no differences in behavior were observed. These data suggest a long-term change in circuital plasticity may be maintained by $\operatorname{Prl}$ after consolidation of the task. On the other hand, it is important to emphasize that no significant differences between groups were observed when analyzing the PD of the gamma frequency band. This should not be surprising, since the role of $\gamma$ oscillations $(30-120 \mathrm{~Hz})$ has been related to memory trace replay and consolidation of information (Eschenko et al., 2008; Girardeau et al., 2009; van de Ven et al., 2016), whereas $\theta$ oscillations (4-12 Hz) are fundamental in locomotion and spatial memory tasks (Buzsáki, 2002; Korotkova et al., 2018). In agreement with this, we showed that $\theta$ PD was not linearly associated to mouse locomotion speed, thus it is feasible that the difference in $\theta$ oscillations was due to an improvement to the learning process in Prl-treated mice. Complementary to the results discussed above, a more detailed analysis during arrival to the target revealed that there was a 7-9 $\mathrm{Hz}$ shift on D2 relative to D1 in both Vhand Prl-treated animals, which was maintained during rest only in Prl-treated mice. We interpreted this change as a faster modification in the $\theta$ oscillation band that was necessary to consolidate the spatial learning in the acquisition phase (D2), coinciding with a decrease in 3-8 $\mathrm{Hz}$ and an increase at $8-12 \mathrm{~Hz}$ in Prl-treated animals. Hippocampal $\theta$ oscillations have been linked to memory processes (Winson, 1978; Buzsaki 2002; McNaughton et al., 2006) and can modulate the 
amplitude of the $\gamma$ rhythm depending on the $\theta$ phase (Bragin et al., 1995). In addition, several studies have associated $\theta-\gamma$ interaction with memory processes (Canolty and Knight, 2010; Lisman and Jensen, 2013; Bott et al., 2016). In line with this experimental evidence, the present data suggest that a tuning in $\theta$ oscillation band without modification to $\gamma$ rhythm is a reasonable correlate of behavioral effects induced by Prl in the acquisition phase of spatial memory tasks.

The hippocampus is an important brain area involved in cognitive functions (Barker and Warburton, 2011), and the present data show that an increase in serum Prl enhanced performance of a spatial memory task. However, information about the effects of Prl in cognition are limited and contradictory. For example, while experimental studies have indicated that pregnancy and motherhood improve learning and memory (Kinsley et al., 1999; Love et al., 2005; Pawluski et al., 2006), clinical evidence indicates a negative linear association between Prl levels and cognitive tests of executive functions in pregnant women during late pregnancy and early postpartum (Henry and Sherwin, 2012). In addition, patients with prolactinomas showed lower scores on memory tasks (Bala et al., 2016) and cognitive deficits in verbal memory and executive function (Yao et al., 2018). In mammals, Prl levels vary across species and sex, as well as in physiological and pathological conditions. Reproductive cycle, pregnancy and lactation are the main causes of physiological hyperprolactinaemia (Freeman et al., 2000; Ben-Jonathan et al., 2008; Phillipps et al., 2020), with varied secretion patterns across different species (Phillipps et al., 2020). On the other hand, pathologies such as pituitary adenoma, renal failure, seizures or drugs including neuroleptics, antiemetics and opioids, among others, can lead to increased serum Prl levels at different concentrations (Samperi et al., 2019). In this scenario, we have shown novel findings indicating that Prl increases spatial learning performance and modifies circuital plasticity in the hippocampus, highlighting its role beyond the classical functions already described. The present data can help us to understand the cognitive changes that occur in both physiological and pathological hyperprolactinemia. One mostly instructive example is the importance of Prl in influencing maternal behavior and promoting modifications in the maternal brain (Bridges et al., 1985; Shingo et al., 2003; Larsen and Grattan, 2010; Brown et al., 2017), which are essential for the survival of offspring (Larsen and Grattan, 2010; Brown et al., 2017). Although the hippocampus has not been classically linked to motherhood, it is clear that spatial learning and memory abilities in maternal care contribute to ensure the normal development and survival of offspring. The mother needs to search for food and water, learn the location of predators and return to nurse the offspring.

\section{Conclusions}

Prl is involved in different brain process beyond the direct physiological functions associated with lactancy and motherhood in mammals. To understand the role of this hormone in physiological and pathological hyperprolactinemia, it was important to elucidate the circuital mechanisms that underlie the effects of Prl at the cognitive level. Here we have provided novel evidence about the long-term circuital changes in the hippocampal oscillatory activity, essential in the acquisition and consolidation processes of spatial learning and memory in mammals, which are critical for species survival.

\section{Author contributions}

BMR contributed to the design of the study, acquisition of data, and analysis and interpretation of data. SM contributed to immunohistochemistry and ELISA studies, AZM and ALA to analysis and interpretation of data. BMR, SM, AZM and ALA revised the manuscript critically for intellectual content. EDM contribute to conceptualization, data analysis and interpretation, write the original draft and revised the manuscript. All authors approved the final version to be published, and agreed to be accountable for all the aspects of the work, ensuring that questions related to the accuracy or integrity are appropriately investigated and resolved.

\section{Funding}

This work was supported by Ministerio de Economía y Competitividad, Ministerio de Ciencia e Innovación, Agencia Estatal de Investigación (Spain) and Fondo Europeo de Desarrollo Regional, UE, to E.D.M (grant BFU2014-57929-P; MINECO/FEDER, UE and BFU201788393-P; AEI/FEDER, UE).

\section{Availability of data and materials}

The data that support of this study are available from the corresponding author, EDM, upon reasonable request.

\section{Declaration of Competing Interest}

None.

\section{Acknowledgements}

The professional editing service NB Revisions was used for technical preparation of the text prior to submission.

\section{References}

Bala, A., Łojek, E., Marchel, A., 2016. Cognitive functioning of patients with a PRLsecreting pituitary adenoma: a preliminary report. Neurology $86,731-734$. https:// doi.org/10.1212/WNL.0000000000002252.

Barker, G.R.I., Warburton, E.C., 2011. When is the hippocampus involved in recognition memory? J. Neurosci. 31, 10721-10731. https://doi.org/10.1523/ JNEUROSCI.6413-10.2011.

Barnes, C.A., 1979. Memory deficits associated with senescence: a neurophysiological and behavioral study in the rat. J. Comp. Physiol. Psychol. 93, 74-104. https://doi. org/10.1037/h0077579.

Bender, F., Gorbati, M., Cadavieco, M.C., Denisova, N., Gao, X., Holman, C., Korotkova, T., Ponomarenko, A., 2015. Theta oscillations regulate the speed of locomotion via a hippocampus to lateral septum pathway. Nat. Commun. 6, 8521. https://doi.org/10.1038/ncomms9521.

Ben-Jonathan, N., LaPensee, C.R., LaPensee, E.W., 2008. What can we learn from rodents about prolactin in humans? Endocr. Rev. 29, 1-41. https://doi.org/10.1210/ er. 2007-0017.

Bole-Feysot, C., Goffin, V., Edery, M., Binart, N., Kelly, P.A., 1998. Prolactin (PRL) and its receptor: actions, signal transduction pathways and phenotypes observed in PRL receptor knockout mice. Endocr. Rev. 19, 225-268. https://doi.org/10.1210/ edrv.19.3.0334.

Bott, J.-B., Muller, M.-A., Jackson, J., Aubert, J., Cassel, J.-C., Mathis, C., Goutagny, R., 2016. Spatial reference memory is associated with modulation of theta-gamma coupling in the dentate gyrus. Cereb. Cortex 26, 3744-3753. https://doi.org/ 10.1093/cercor/bhv177.

Bragin, A., Jando, G., Nadasdy, Z., Hetke, J., Wise, K., Buzsaki, G., 1995. Gamma (40-100 Hz) oscillation in the hippocampus of the behaving rat. J. Neurosci. 15, 47-60. https://doi.org/10.1523/JNEUROSCI.15-01-00047.1995.

Bridges, R.S., Grattan, D.R., 2019. 30 years after: CNS actions of prolactin: sources, mechanisms and physiological significance. J. Neuroendocrinol. 2019, e12669. https://doi.org/10.1111/jne.12669.

Bridges, R.S., DiBiase, R., Loundes, D.D., Doherty, P.C., 1985. Prolactin stimulation of maternal behavior in female rats. Science 227, 782-784. https://doi.org/10.1126/ science.3969568.

Brown, R.S.E., Aoki, M., Ladyman, S.R., Phillipps, H.R., Wyatt, A., Boehm, U., Grattan, D. R., 2017. Prolactin action in the medial preoptic area is necessary for postpartum maternal nursing behavior. Proc. Natl. Acad. Sci. U. S. A. 114, 10779-10784. https://doi.org/10.1073/pnas.1708025114.

Buzsáki, G., 2002. Theta oscillations in the hippocampus. Neuron 33, 325-340. https:// doi.org/10.1016/s0896-6273(02)00586-x.

Cabrera-Reyes, E.A., Limón-Morales, O., Rivero-Segura, N.A., Camacho-Arroyo, I., Cerbón, M., 2017. Prolactin function and putative expression in the brain. Endocrine 57, 199-213. https://doi.org/10.1007/s12020-017-1346-x.

Canolty, R.T., Knight, R.T., 2010. The functional role of cross-frequency coupling. Trends Cogn. Sci. 14, 506-515. https://doi.org/10.1016/j.tics.2010.09.001.

Eschenko, O., Ramadan, W., Mölle, M., Born, J., Sara, S.J., 2008. Sustained increase in hippocampal sharp-wave ripple activity during slow-wave sleep after learning. Learn. Mem. 15, 222-228. https://doi.org/10.1101/lm.726008.

Freeman, M.E., Kanyicska, B., Lerant, A., Nagy, G., 2000. Prolactin: structure, function, and regulation of secretion. Physiol. Rev. 80, 1523-1631. https://doi.org/10.1152/ physrev.2000.80.4.1523. 
Girardeau, G., Benchenane, K., Wiener, S.I., Buzsáki, G., Zugaro, M.B., 2009. Selective suppression of hippocampal ripples impairs spatial memory. Nat. Neurosci. 12, 1222-1223. https://doi.org/10.1038/nn.2384.

Grattan, D.R., Kokay, I.C., 2008. Prolactin: a pleiotropic neuroendocrine hormone. J. Neuroendocrinol. 20, 752-763. https://doi.org/10.1111/j.13652826.2008.01736.x.

Henry, J., Sherwin, B., 2012. Hormones and cognitive functioning during late pregnancy and postpartum: a longitudinal study. Behav. Neurosci. 126, 73-85. https://doi.org/ $10.1037 / \mathrm{a} 0025540$.

Kinsley, C.H., Madonia, L., Gifford, G.W., Tureski, K., Griffin, G.R., Lowry, C., Williams, J., Collins, J., McLearie, H., Lambert, K.G., 1999. Motherhood improves learning and memory. Nature 402, 137-138. https://doi.org/10.1038/45957.

Korotkova, T., Ponomarenko, A., Monaghan, C.K., Poulter, S.L., Cacucci, F., Wills, T., Hasselmo, M.E., Lever, C., 2018. Reconciling the different faces of hippocampal theta: the role of theta oscillations in cognitive, emotional and innate behaviors. Neurosci. Biobehav. Rev. 85, 65-80. https://doi.org/10.1016/j. neubiorev.2017.09.004.

Larsen, C.M., Grattan, D.R., 2010. Prolactin-induced mitogenesis in the subventricular zone of the maternal brain during early pregnancy is essential for normal postpartum behavioral responses in the mother. Endocrinology 151, 3805-3814. https://doi. org/10.1210/en.2009-1385.

Leem, Y.H., Park, J.S., Chang, H., Park, J., Kim, H.S., 2019. Exercise prevents memory consolidation defects via enhancing prolactin responsiveness of CA1 neurons in mice under chronic stress. Mol. Neurobiol. 56, 6609-6625. https://doi.org/10.1007/ s12035-019-1560-z.

Lisman, J.E., Jensen, O., 2013. The theta-gamma neural code. Neuron 77, 1002-1016. https://doi.org/10.1016/j.neuron.2013.03.007.

Love, G., Torrey, N., McNamara, I., Morgan, M., Banks, M., Hester, N.W., Glasper, E.R., Devries, A.C., Kinsley, C.H., Lambert, K.G., 2005. Maternal experience produces long-lasting behavioral modifications in the rat. Behav. Neurosci. 119, 1084-1096. https://doi.org/10.1037/0735-7044.119.4.1084.

Martin, S.J., Grimwood, P.D., Morris, R.G.M., 2000. Synaptic plasticity and memory: an evaluation of the hypothesis. Annu. Rev. Neurosci. 23, 649-711. https://doi.org/ 10.1146/annurev.neuro.23.1.649.

McNaughton, N., Ruan, M., Woodnorth, M.A., 2006. Restoring theta-like rhythmicity in rats restores initial learning in the Morris water maze. Hippocampus 16, 1102-1110. https://doi.org/10.1002/hipo.20235.

Nicoll, R.A., 2017. A brief history of long-term potentiation. Neuron 93, 281-290. https://doi.org/10.1016/j.neuron.2016.12.015.
Pawluski, J.L., Walker, S.K., Galea, L.A., 2006. Reproductive experience differentially affects spatial reference and working memory performance in the mother. Horm. Behav. 49, 143-149. https://doi.org/10.1016/j.yhbeh.2005.05.016.

Paxinos, G., Franklin, K.B.J., 2012. In: Elsevier, B.V. (Ed.), Paxinos and Franklin's the Mouse Brain in Stereotaxic Coordinates, fourth ed.

Phillipps, H.R., Yip, S.H., Grattan, D.R., 2020. Patterns of prolactin secretion. Mol. Cell. Endocrinol. 502, 110679 https://doi.org/10.1016/j.mce.2019.110679.

Rivas, J., Gaztelu, J.M., García-Austt, E., 1996. Changes in hippocampal cell discharge patterns and theta rhythm spectral properties as a function of walking velocity in the guinea pig. Exp. Brain Res. 108, 113-118. https://doi.org/10.1007/BF00242908.

Samperi, I., Lithgow, K., Karavitaki, N., 2019. Hyperprolactinaemia. J. Clin. Med. 8, 2203. https://doi.org/10.3390/jcm8122203.

Segal, M., 2017. Dendritic spines: morphological building blocks of memory. Neurobiol. Learn. Mem. 138, 3-9. https://doi.org/10.1016/j.nlm.2016.06.007.

Shingo, T., Gregg, C., Enwere, E., Fujikawa, H., Hassam, R., Geary, C., Cross, J.C., Weiss, S., 2003. Pregnancy-stimulated neurogenesis in the adult female forebrain mediated by prolactin. Science 299, 117-120. https://doi.org/10.1126/ science. 1076647.

Sunyer, B., Patil, S., Höger, H., Luber, G., 2007. Barnes maze, a useful task to assess spatial reference memory in the mice. Protoc. exch. https://doi.org/10.1038/ nprot.2007.390.

Torner, L., Tinajero, E., Lajud, N., Quintanar-Stéphano, A., Olvera-Cortés, E., 2013. Hyperprolactinemia impairs object recognition without altering spatial learning in male rats. Behav. Brain Res. 252, 32-39. https://doi.org/10.1016/j. bbr.2013.05.031.

van de Ven, G.M., Trouche, S., McNamara, C.G., Allen, K., Dupret, D., 2016. Hippocampal offline reactivation consolidates recently formed cell assembly patterns during sharp wave-ripples. Neuron 92, 968-974. https://doi.org/10.1016/j. neuron.2016.10.020.

Walker, T.L., Vukovic, J., Koudijs, M.M., Blackmore, D.G., Mackay, E.W., Sykes, A.M. Overall, R.W., Hamlin, A.S., Bartlett, P.F., 2012. Prolactin stimulates precursor cells in the adult mouse hippocampus. PLoS One 7, e44371. https://doi.org/10.1371/ journal.pone.0044371.

Winson, J., 1978. Loss of hippocampal theta rhythm results in spatial memory deficit in the rat. Science 201, 160-163. https://doi.org/10.1126/science.663646.

Yao, S., Song, J., Gao, J., Lin, P., Yang, M., Zahid, K.R., Yan, Y., Cao, C., Ma, P., Zhang, H., Li, Z., Huang, C., Ding, H., Xu, G., 2018. Cognitive function and serum hormone levels are associated with gray matter volume decline in female patients with prolactinomas. Front. Neurol. 8, 742. https://doi.org/10.3389/ fneur.2017.00742. 